\title{
Immature Ovarian Teratoma in a 21 Year-Old Woman: A Case Report and Review of the Literature
}

Sofoklis Stavrou*, Ekaterini Domali , loannis Paraoulakis , Dimitrios Haidopoulos ,Nikolaos Thomakos , Dimitrios Loutradis ,Peter Drakakis Department of Obstetrics and Gynecology, University of Athens, Alexandra Hospital, 11528, Athens, Greece

Abbreviations: IOTA: International Ovarian Tumor Analysis; MRI: Magnetic Resonance Imaging; CT: Computed Tomography; 3D: 3 Dimensional; NCCN: National Comprehensive Cancer Network; ECOG: Eastern Cooperative Oncology Group.

\section{Introduction}

Ovarian tumors can be categorized into epithelial, germ cell, sex cord stromal or metastatic ones [1]. The ovarian teratomas are divided into mature teratomas, immature teratomas and monodermal teratomas (like struma ovarii, carcinoid tumors, and neural tumors). They represent the largest part of germ cells neoplasms [1,2]. Mature cystic teratomas (dermoid cysts) constitute the majority of ovarian germ cell tumors accounting for $20 \%$ of all ovarian neoplasms [3]. Struma ovarii tumors represent uncommon ovarian tumors containing completely or mainly thyroid tissue [3]. Immature teratomas differ from mature cystic teratomas because they have clinically malignant behavior , are not as common as mature cystic teratomas ( $<1 \%$ of ovarian teratomas) and are histologically characterized by the presence of immature or embryonic tissues. They affect younger women (frequently for the first 2 decades of life), with crest occurrence between 15 and 19 years old [4,5]. Immature teratomas rarely appear during the menopausal period [5]. This report discusses the case of a 21 year-old female with a large immature teratoma in the right ovary and its diagnostic, histomorphological and surgical modalities.

\section{Case Presentation}

A 21 year-old Greek female, overweight (BMI: 31), gravida 0, parity 0 , with no previous health problems, an age of first menstruation of 12 years, attended our hospital (11/2013), complaining for sustained episodes of diarrhea and feeling of gradually increased abdominal bloating since 48 hours. Clinical evaluation proved increased abdominal size and revealed a palpable mass on the right lower abdomen.

Transvaginal ultrasonography showed a mass of $91 \times 101 \times 100$ $\mathrm{mm}$ dimensions originated from the right ovary. Based on IOTA criteria, applied also in 3Dimages, mass has been described as a multilocular-solid lesion, containing $>5$ loci [6]. The solid part of $75 \times$ $59 \times 77 \mathrm{~mm}$ was completely irregular. More than five irregular papillary projections were calculated. It was notable that 3 of them presented internal flow. Strong adhesions behind the mass were revealed during 3D examination. Vascularization pattern was estimated as excessive, meaning color score IV. Ascites was clearly obvious and the suggestion of "floated uterus" was included in the report. Swollen left iliac lymph nodes were recognized but without clinical significance. Based on the above reported ultrasonographic data and regarding IOTA simple rules $[7,8]$, the ovarian lesion was characterized as a "probably malignant tumor" (Figure 1).

Laboratory results showed that $\mathrm{Ht}, \mathrm{Hb}$, PLT, WBC, Glu, Urea, $\mathrm{Cr}$, total bilirubin, $\mathrm{Na}, \mathrm{K}$, ALP, gamma-GT, CPK, CK-MB, amylase, CRP, bHCG $(<1.20 \mathrm{U} / \mathrm{I})$ were fluctuated in normal ranges. In our Department every single patient with ovarian mass is tested standard

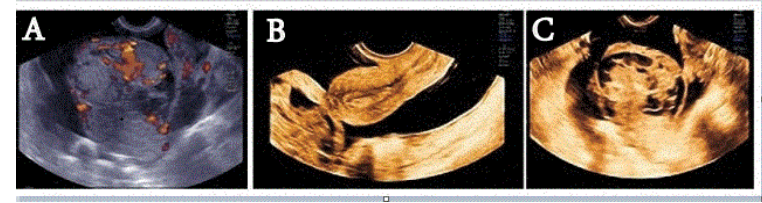

Figure 1: $3 D$ images of ovarian lesion; A: color score IV with centrally distributed vessels; B: uterus surrounded by ascites; C: multilocular-solid mass, containing $>5$ loci and irregular solid part while external wall was appeared interrupted.

with specific tumor markers .So, tumor markers Ca 15-3, Ca19-9, Ca 125 , and CEA were fluctuated in normal ranges. Serum levels of alphafetoprotein (a-FP) were significantly elevated reaching the plateau of $392 \mathrm{iu} / \mathrm{ml}$ (normal ranges: $<9.5 \mathrm{iu} / \mathrm{ml}$ ), supporting the sonographically evoked opinion of underlying malignancy. Except that AST (62 IU/L), ALT (73 IU/L) and LDH (309 IU/L) were mildly increased.

Because of the young profile of the patient, MRI of upper and lower abdomen has been also performed; similarly to ultrasonographic report, MRI noticed the presence of excessive ascites; it also described a heterogeneous right ovarian mass of $88 \times 81 \times 100 \mathrm{~mm}$ presenting cystic, solid and hemorrhagic contents, fixed to the broad ligament of the uterus. It also revealed 2 thin walled cysts of $4,3 \mathrm{~cm}$ and $2,4 \mathrm{~cm}$ in the left ovary without solid parts. Intraperitoneal tissue in the region of upper abdomen was described, implying the possibility of underlying peritoneal carcinomatosis. The liver, the biliary system, the spleen, the pancreas and the adrenals were normal. MRI didn't reveal enlarged paraaortic, mesenteric, iliac or inguinal lymph nodes (Figure 2).

Chest X-ray and ultrasonographically breast examination were normal. Initially, ascites suction was realized in order to alleviate from pressing symptoms. In the operation theater under general anesthesia, laparoscopy was performed with the technique of the four ports for better abdominal exploration in the overweight patient; 4 to 5 liters of ascites were aspirated for cytological examination. The organs of the upper abdomen were normal, without implantations in the peritoneum or omental disease. The uterus and the left adnexa were normal but a mass 10-12 cm of right ovary characterized by abnormal surface was revealed. So exploratory laparotomy followed with vertical incision. Then the cystic lesion was removed and sent for frozen section. The rapid biopsy was positive for

${ }^{*}$ Corresponding author: Sofoklis Stavrou, University of Athens, Alexandra Hospital, 11528, Athens, Greece, E-mail: sfstavrou@yahoo.com

Received January 28, 2016; Accepted February 09, 2016; Published February 17,2016

Citation: Stavrou S Domali E, Paraoulakis I, Haidopoulos D, Thomakos N, et al (2016) Immature Ovarian Teratoma in a 21 Year-Old Woman: A Case Report and Review of the Literature. J Gen Pract 4: 232. doi: 10.4172/2329-9126.1000232

Copyright: ( 2016 Stavrou S. This is an open-access article distributed under the terms of the Creative Commons Attribution License, which permits unrestricted use, distribution, and reproduction in any medium, provided the original author and source are credited. 


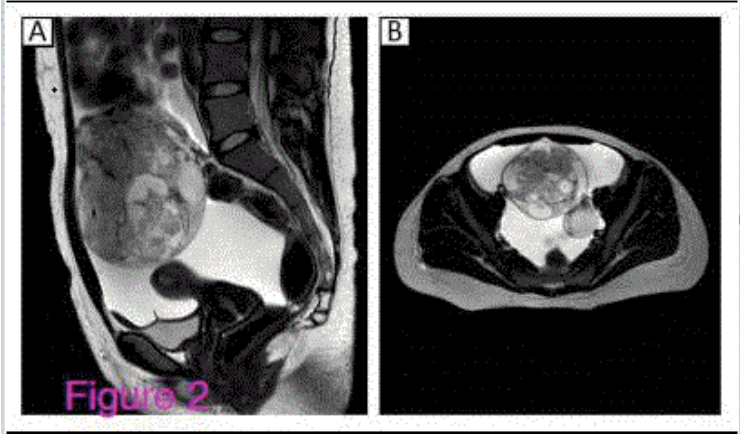

Figure 2: Magnetic resonance imaging (MRI); $A$ : T2W sagittal and $B$ : T2Wtransverse reformation showing a mass of $88 \times 81 \times 100 \mathrm{~mm}$ dimensions, originated from the right ovary.

malignancy. The lab evoked the diagnosis of immature teratoma vs yolk salk tumor. Based on the results, we proceeded to a right adnexectomy with the infundibulopelvic ligament followed by omentectomy; even though there is no consensus for a systematic lymphadenectomy in germ cell tumors [9], bilateral iliac and paraaortic lymphadenectomy as well as appendectomy were performed. The operation was terminated by the washing of the peritoneal cavity and the placement of drainage. The patient stood the procedure well and the postoperative period was uneventful.

Finally, histopathological report supported by immunochemistry, reinforced the diagnosis of grade III ovarian immature teratoma which infiltrated and disintegrated the capsule. The mass contained immature neuroepithial tissue that formed rosettes, intestinal type epithelial tissue, cartilage and vascular formations. There were also foci of necrosis as well as hemorrhagic loci. The total of 27 excised lymph nodes were tumor free. The immunochemistry using P53 and K67 was positive for AFP. The histopathologist didn't use for the diagnosis assessment the karyotyping because in our Department, karyotyping isn't used as a standard routine diagnostic tool because chromosomal abnormalities had been only revealed in about 20 patients the last 20 years based on our histopathological archives (Figure 3).

Based on the above reported data, oncologist's council proposed that this patient affected by stage Ic, grade III ovarian immature teratoma and should be further treated by chemotherapy. The patient was referred to the Clinical Oncology Department for adjuvant chemotherapy (12/2013) and received 3 cycles of Bleomycin/Etoposide/Cisplatin (BEP) plus GCSF for five days in each cycle. After the last cycle (3/2014) the patient was followed up 1 month later (4/2014) with normal levels of Ca-125(7 U/ $\mathrm{mL}), \mathrm{B}-\mathrm{HCG}(1 \mathrm{IU} / \mathrm{L})$ and AFP (4.6 IU/mL). Two months later $(6 / 2014)$ the patient was clinically and ultrasonographically normal .In addition the markers were in normal ranges B-HCG (2 IU/L) and AFP $(3.2 \mathrm{IU} / \mathrm{mL})$. Her menstrual cycles started to be regular (monthly) within 3 months after the last application of chemotherapy (6/2014). Totally 6 months after the last cycle of Chemotherapy (9/2014) the patient was asymptomatic in the clinical examination and the markers were ranged in regular levels $\mathrm{Ca}-125$ : $3 \mathrm{U} / \mathrm{mL}$, B- HCG: 4 IU/Land AFP: $3 \mathrm{IU} / \mathrm{mL}$. The transvaginal ultrasonic image was exactly the same as last time without new findings, as well as her menstrual cycle was normal. The patient until now and for 5 years after the last cycle of chemotherapy is followed up with the NCCN guidelines for malignant germ cell tumors .Her performance status based to ECOG criteria is grade 0 each time she visits our hospital for her follow up.
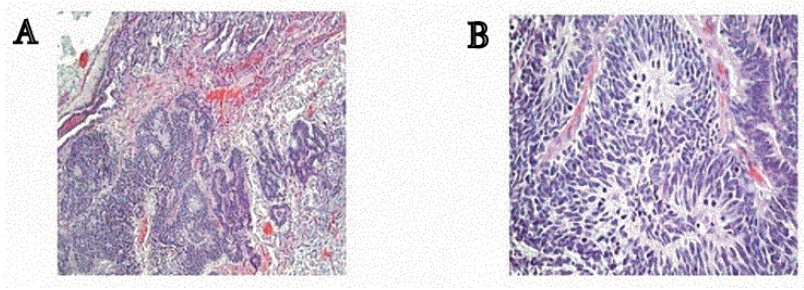

Figure 3: A. Immature embryonal-type tissue which consists mainly by tubules and rosettes of neuroectodermal tissue in loose myxoid stroma (H/E X100) B. The neuroepithelial rosettes present numerous mitotic figures (H/E X 400).

\section{Discussion}

Ovarian cancer occurs rarely in children and adolescents; it has an estimated annual rate of 2.6 cases per 100,000 girls per year. The word teratoma derives from the Greek word "teras" (monster) [2]. Teratomas are mainly identified in young women, $<45 \mathrm{yrs}$, arising from the ovary [1]. It should be noticed that they consist almost the half of the malignant tumors observed in infancy [10]. Rarely, they might originate from brain, retroperitoneum, pericardium and organs like thyroid, liver, parotid gland, breast, stomach, bladder and esophagus $[1,2]$. Based on APO reports, teratomas are discriminated in benign mature and malignant immature pathologies. Malignant teratomas represent $3 \%$ of teratomas, $1 \%$ of ovarian cancer and $20 \%$ of malignant ovarian germ cell tumors [2]. Other forms of malignant germ cell tumors are dysgerminoma and endodermal sinus tumors (EST). Embryonal carcinoma, polyembryoma end choriocarcinoma consist a very rare form of germ cell tumors. Another type of tumor which can be presented is malignant mixed germ cell tumor [11].

Malignant teratomas are described as immature or monodermal lesions such as struma ovarii in 5\% of the cases, carcinoid masses and neural cancers [3]. In a recent Tai study of 76 patients, Neeyalavira showed that the most common histology of malignant ovarian germ cell tumors was immature teratomas (34.2\%). Nevertheless according to Smith, rates for immature teratomas are higher for Asian/Pacific Islanders and Hispanics than Caucasians [12,13]. More specifically, immature formations of teratomas are classified into three categories: firstly, lesions that include immature ectodermic tissue and neuroectodermal epithel in rosettes and tubules, secondly, masses that contain immature mesenchyme with immature cartilage, immature fat, osteoids and rhabdomyoblasts and finally immature endodermal tissue as well as hepatic tissue, intestinal type epithel with basal vacuolization and embryonic renal tissue [1]. Macroscopically immature teratomas are larger $(14-25 \mathrm{~cm})$ than cystic teratomas $(\max$ diameter: $7 \mathrm{~cm}$ approximately). They can be solid or solid with cystic components. Cystic areas contain serous fluid, mucinous fluid or fat elements [4].

They are mainly discovered during annual routine examination via ultrasonography or as an ovarian lesion complicated in urgent cases. More specifically, teratomas could also present more frequently torsion (3.2-16\%), rupture or rarely malignant deterioration 0.17 $2 \%)$. Squamous cell carcinoma that arises from the ectoderm has been found to be responsible for malignant transformation [14]. Contrarily, malignant meaning immature and monodermal teratomas express immediately clinically malignant behavior. Despite the fact that they are rarely identified, they consist a really hazardous lesion. Patients usually complain of pelvic pain, swollen abdomen, diarrhea, constipation and abnormal uterine bleeding. Further observed symptoms are defined by 
the spread of the disease; the last could be peritoneum or retroperitoneal lymph nodes as well as an extremely rarely hematogenous dissemination to lungs, brain or liver [5]. Diagnosis is mainly based on imaging and secondary in blood tests. In young patients, human chorionic gonadotropin (HCG), $\alpha$-fetoprotein (AFP) and lactate dehydrogenase (LDH), complete blood count, and liver and renal function tests should be carried out [9].

Ultrasonography, as the first line examination, plays a pivotal role in the preoperative discrimination of mature teratomas based on pattern recognition. 3D ultrasonography leads in more clarified images based also on pattern recognition.

Imaging of solid hyperechogenic smooth masses that affect part or the entire structure of the ovary combined to acoustic shadows imply the diagnosis of mature teratomas; acoustic shadows consist strong shadows produced by the content of the mass as well as the surrounded intestinal loops [15]. They could also be presented as mixed lesions consisting of a multiplicity of loci constructed by fine, horizontally orientated, hyperechogenic sparkling lines and/or a hyperechogenic solid part. Accumulation of hair and sebaceous fat tissue leads in the ultrasonographic reported appearance of white lines and hyperechogenic solid formations that characterize mature teratomas $[16,17]$ (Figure 4).

Rokitansky protuberance as well as white dots and/or white balls that could be attributed to the presence of sebaceous serous tissue, hair or calcified tissues such as bone and/or teeth, as ultrasonographic discriminator markers are usually observed [10,18,19] (Figure 5).

Unusual features that do not follow the above reported patterns recognition have been identified (Figures 6 and 7).

Finally, it has been reported that mature cystic teratomas are poorly vascularized lesions. It must be noticed that vascularization, if obvious, is restricted across the periphery of the structure [20].

On the other hand, immature teratomas show mixed echogenicity caused by the coexistence of cystic and solid parts. Presence of irregular and non continuous external wall, multilocularity of the lesion as well as identification of irregular, vascularized papillary projections should imply the probability of malignancy; based on IOTA pattern recognition imaging of color score IV vascularization model as well as centrally distributed vessels inside the mass should reinforce the examiner to suspect the existence of possibility of malignancy $[3,4,6]$.

$\mathrm{CT}$ and MRI are considered as specific imaging examinations that offer in the exact analysis and characterization of ovarian lesions; in cases of teratomas, both exams lead in the production of images similar to ultrasonographic products; therefore it would be suggested that they could react only supplementary in the doubtful cases [3].
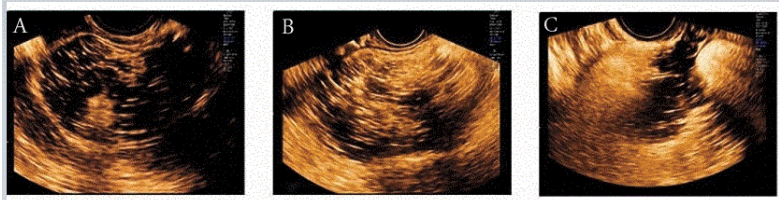

Figure 4: 3D imaging of mature teratomas; fine hyperechogenic sparkled lines that cross the entire ovary (A and B); mixed echogenicity evoked by solid hyperechogenic smooth part, acoustic shadows and white lines and dots (C).
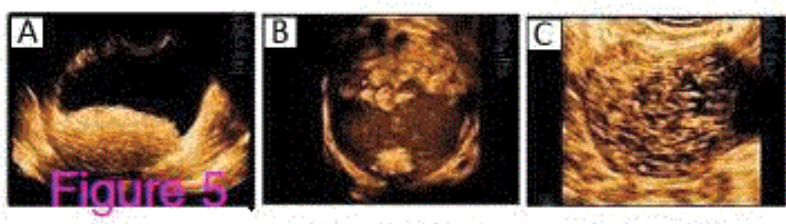

Figure 5: 3D images where Rokitansky perturbation is observed surrounded by anechoic fluid (A); internal 4D imaging of mature teratoma appearance where white formations, and fat fluid are obvious (B); 3D presentation of ovarian mass characterized predominantly by the accumulation of white calcified dots $(\mathrm{C})$.
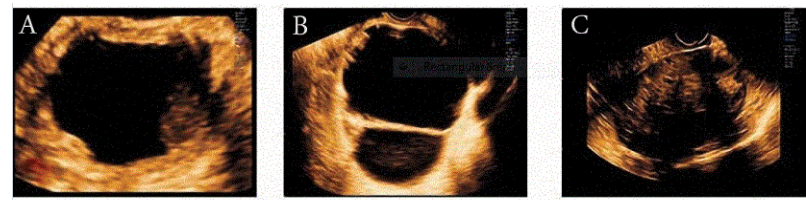

Figure 6: 3D images of ovarian lesions that have been histologically proven to be mature cystic teratomas; $\mathrm{A}$ : unilocular mass with an irregular papillary projection; $\mathrm{B}$ : bilocular mass of low level echogenicity, regular hyperechogenic septum and irregular wall; C: unilocular-solid mass where solid part is irregular.
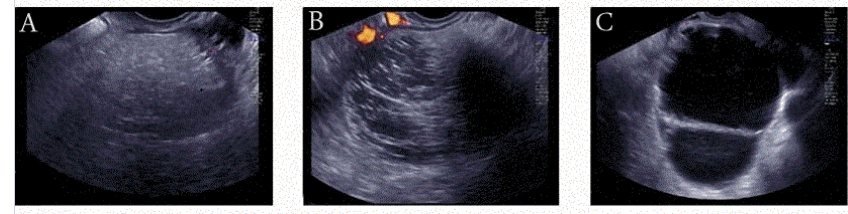

Figure 7: 3D images of poorly vascularized mature cystic teratomas of typical $(A, B)$ or atypical $(C)$ features based on pattern recognition's characteristics.

Immature teratomas mainly occur young women; so the preservation of fertility in these patients plays a pivotal role for the management. Patients who belong to grade 1 and FIGO stage 1 tumors are treated by staging surgery with unilateral oophorectomy [21].

Chemotherapy is suggested in patients affected by tumors characterized by APO reports as grade 2 and 3 . In these cases, adjuvant chemotherapy includes bleomycin, cisplatin and etoposide adding up to surgery is proposed $[9,21]$. Fertility profile is not influenced in young patients that undergo chemotherapy after operation realization. Even after chemotherapy at least $80 \%$ of patients can resume normal cycle. Fertility profile is only affected in cases of multidrug combination chemotherapy before or after the operation theater. These patients have a reduced number of primordial follicles and an augmentation in stromal fibrosis in atrophied cortices; the latter leads in increase in serum gonadotropins levels and simultaneously decrease in estradiol levels. This hormonal disorganization combined with other factors such as type of chemotherapy, high doses and duration of chemotherapy, appears to affect the function of the ovaries [22]. It has been showed that radiotherapy does not advance the prognosis [5]. The prognosis of immature teratomas is based on FIGO staging and is influenced by factors like grade, growth rate, rupture of the capsule and lymphovascular infiltration [2]. Bilateral immature teratoma represents an uncommon 
Citation: Stavrou S Domali E, Paraoulakis I, Haidopoulos D, Thomakos N, et al. (2016) Immature Ovarian Teratoma in a 21 Year-Old Woman: A Case Report and Review of the Literature. J Gen Pract 4: 232. doi: 10.4172/2329-9126.1000232

situation, accounting for $10 \%$ of cases. Bilateral immature teratomas have a five-year survival rate of $80.7 \%$ opposite the survival rate of unilateral tumors, accounting for $93.6 \%$ [5]. It is widely accepted that ovarian cancer incidences should be eliminated at the level of zero; so we think that no case should be lost.

\section{Conclusion}

Malignancy in women $<20$ yrs old is confined between 0.2 and 1.4 per100.000 while in women between 20-29 yrs old does not exceed the level of 2.2 per 100.000 . Based on this rare occurrence, we think that each case of immature teratoma should be presented and discussed. Ultrasonography is the first choice imaging for assessing a pelvic mass. The simple IOTA rules have an accuracy of $76-89 \%$ for the diagnosis of ovarian cancer [23]

\section{Authors' Contributions}

The corresponding author SS planned and designed this case report, participated in data collection and interpretation, prepared the manuscript by searching the literature which has been analyzed. DE was the experienced ultrasonographer who made the ultrasounds of the patient based on IOTA pattern before and after the surgery and during her follow up. Except of that DE participated in the preparation of the manuscript and the literature analysis. HD and TN were the surgeons of the patient who gived us the details of the surgery and explained us the surgical procedure .PI participated in data collection. LD is the professor of our hospital and participated in the preparation of this manuscript through his great gynecological experience .Finally DP, associate professor of our hospital had an important and main role of this manuscript, because he helped the corresponding author not only to collect information and design the case report but also he stood next the patient encouraging her.

\section{Acknowledgements}

There is no source of funding for this case report.

\section{References}

1. Saba L, Guerriero S, Sulcis R, Virgilio B, Melis G, et al. (2009) Mature and immature ovarian teratomas: CT, US and MR imaging characteristics. Eur J Radiol 72: 454-463.

2. Gheorghisan-Galateanu A, Terzea DC, Carsote M, Poiana C (2013) Immature ovarian teratoma with unusual gliomatosis. J Ovarian Res 6: 28

3. Savelli L, Testa AC, Timmerman D, Paladini D, Ljungberg O, et al. (2008) Imaging of gynecological disease (4): clinical and ultrasound characteristics of struma ovarii. Ultrasound Obstet Gynecol 32: 210-219.

4. Outwater EK, Siegelman ES, Hunt JL (2001) Ovarian teratomas: tumor types and imaging characteristics. Radiographics 21: 475-490.

5. Barbosa LC, Francisco AM, Archângelo Sde C, Soares FC, Ferreira MC, et al (2012) Bilateral immature ovarian teratoma in a 12-year-old girl: case report. Rev Assoc Med Bras 58: 138-140.

6. Timmerman D, Valentin L, Bourne TH, Collins WP, Verrelst H, et al. (2000) International Ovarian Tumor Analysis (IOTA) Group.Terms, definitions and measurements to describe the sonographic features of adnexal tumors: a consensus opinion from the International Ovarian Tumor Analysis (IOTA) Group. Ultrasound Obstet Gynecol 16: 500-505.
7. Timmerman D, Ameye L, Fischerova D, Epstein E, Melis GB, et al. (2010) Simple ultrasound rules to distinguish between benign and malignant adnexal masses before surgery: prospective validation by IOTA group. BMJ 341: c6839.

8. Kaijser J, Bourne T, Valentin L, Sayasneh A, Van Holsbeke C, et al. (2013) Improving strategies for diagnosing ovarian cancer: a summary of the International Ovarian Tumor Analysis (IOTA) studies. Ultrasound Obstet Gynecol 41: 9-20.

9. Colombo N, Peiretti M, Garbi A, Carinelli S, Marini C et al. (2012) ESMO Guidelines Working Group. Non-epithelial ovarian cancer: ESMO Clinical Practice Guidelines for diagnosis, treatment and follow-up. Ann Oncol 7: 20-26.

10. Damarey B, Farine M, Vinatier D, Collinet P, Lucot J, et al. (2010) [Mature and immature ovarian teratomas: US, CT and MR imaging features]. J Radiol 91 $27-36$

11. Goyal LD, Kaur S, Kawatra K (2014) Malignant mixed germ cell tumour of ovary--an unusual combination and review of literature. J Ovarian Res 7: 91.

12. Smith HO, Berwick M, Verschraegen CF, Wiggins C, Lansing L, et al. (2006) Incidence and survival rates for female malignant germ cell tumors. Obstet Gynecol 107: 1075-1085.

13. Neeyalavira V, Suprasert $P$ (2014) Outcomes of malignant ovarian germ-cel tumors treated in Chiang Mai University Hospital over a nine year period. Asian Pac J Cancer Prev 15: 4909-4913.

14. Petousis S, Kalogiannidis I, Margioula-Siarkou C, Traianos A, Miliaras D, et al. (2013) Mature ovarian teratoma with carcinoid tumor in a 28-year-old patient Case Rep Obstet Gynecol 2013: 108582.

15. Sheth S, Fishman EK, Buck JL, Hamper UM, Sanders RC (1988) The variable sonographic appearances of ovarian teratomas: correlation with CT. AJR Am J Roentgenol 151: 331-334.

16. Guerriero S, Ajossa S, Garau N, Piras B, Paoletti AM, et al. (2005) Ultrasonography and color Doppler-based triage for adnexal masses to provide the most appropriate surgical approach. Am J Obstet Gynecol 192: 401-406.

17. Chen TC, Kuo HT, Shyu SK, Chu CP, Chang TC (2011) Endometriosis coexisting with dermoid cyst in a single ovary: a case report. Chin Med J (Engl) 124: $627-630$.

18. Altinbas SK, Yalvac S, Kandemir O, Altinbas NK, Karcaaltincaba D, et al. (2010) An unusual growth of ovarian cystic teratoma with multiple floating balls during pregnancy: a case report. J Clin Ultrasound 38: 325-327.

19. Buy JN, Ghossain MA, Moss AA, Bazot M, Doucet M, et al. (1989) Cystic teratoma of the ovary: CT detection. Radiology 171: 697-701.

20. Zalel Y, Seidman DS, Oren M, Achiron R, Gotlieb W, et al. (2000) Sonographic and clinical characteristics of struma ovarii. J Ultrasound Med 19: 857-861.

21. Deodhar KK, Suryawanshi P, Shah M, Rekhi B, Chinoy RF (2011) Immature teratoma of the ovary: a clinicopathological study of 28 cases. Indian J Patho Microbiol 54: 730-735.

22. Anita AN, Rushdan MN (2012) Reproductive function after treatment of ovarian germ cell malignancy. Med J Malaysia 67: 71-76.

23. Nunes N, Ambler G, Foo X, Naftalin J, Widschwendter M, et al. (2014) Use of IOTA simple rules for diagnosis of ovarian cancer: meta-analysis. Ultrasound Obstet Gynecol 44: 503-514. 